\title{
Microvascular abnormalities secondary to radiation therapy in neovascular age-related macular degeneration: findings from the INTREPID clinical trial
}

\author{
Florentina Joyce Freiberg, ${ }^{1}$ Stephan Michels, ${ }^{1,2}$ Alyson Muldrew, ${ }^{3}$ Jason Slakter, ${ }^{4}$ \\ Denis $0^{\prime}$ Shaughnessy, ${ }^{5}$ Alan Czeszynski, ${ }^{6}$ Linda Danielson, ${ }^{7}$ Timothy L Jackson, ${ }^{8}$ \\ Usha Chakravarthy ${ }^{9}$
}

${ }^{1}$ Department of Ophthalmology, City Hospital Triemli, Zurich, Switzerland

${ }^{2}$ Augenklinik, University of Zurich, Zurich, Switzerland ${ }^{3}$ Central Angiographic Resource Facility, Queen's University of Belfast, Belfast, UK ${ }^{4}$ Retina Vitreous Consultants of New York, Digital Angiography Reading Center, New York City, New York, USA

${ }^{5}$ Clinical Development, Aura, 85 Bolton Street, Cambridge, Massachussetts, USA ${ }^{6}$ Department of Research and Development, Neurovision Imaging LLC, 1395 Garden Highway, Sacramento, California 95833, USA

${ }^{7}$ The International Drug Development Institute, Louvainla-Neuve, Belgium

${ }^{8}$ Ophthalmology, King's College London, London, UK

${ }^{9}$ Centre for Experimental Medicine, Queen's University of Belfast, Belfast, UK

\section{Correspondence to}

Dr Usha Chakravarthy, Center for Experimental Medicine, Queen's University of Belfast, Belfast BT12 6BA, UK; u. chakravarthy@qub.ac.uk

Received 5 January 2018 Accepted 25 May 2018 Published Online First 21 June 2018

\section{ABSTRACT \\ Purpose To report the incidence and features of retinal microvascular abnormalities (MVAs) occurring secondary to stereotactic radiotherapy (SRT) in a randomised double-masked sham-controlled clinical trial at 21 European sites.}

Methods Two hundred and thirty participants with neovascular age-related macular degeneration (AMD) treated with at least three intravitreal antivascular endothelial growth factor (anti-VEGF) injections prior to enrolment, and demonstrating a continuing need for re-treatment. Interventions: 16 Gy, 24 Gy or sham SRT. All three groups received pro re nata anti-VEGF injections if the lesion was judged to be active at review visits. Colour fundus images from baseline and 6 months and fluorescein angiograms from baseline and annual visits were graded for measures of morphological outcome and safety using a prespecified protocol with accompanying definitions to distinguish RT-related MVA from non-specific retinal vessel abnormalities that are known to occur in neovascular AMD. The main outcome measure was MVA detected by months 12, 24 and 36 after enrolment.

Results The frequency of MVAs in the combined SRT arms was $0 \%$ in year $1,13.1 \%$ in year 2 and $30.3 \%$ in year 3. The area of MVA was small and the mean change in visual acuity in year 2 was similar in a subset of SRT eyes with MVAs, versus those without MVAs. MVA was considered to have possibly contributed to vision loss in 2 of 18 cases with MVA in year 2, and 5 of 37 cases in year 3 .

Conclusion Treatment with SRT is associated with development of subtle MVAs that have little or no impact on visual outcome. These findings can help clinicians recognise the retinal MVAs that occur in response to SRT.

\section{INTRODUCTION}

A recent randomised controlled trial that enrolled patients who were chronically dependent on antivascular endothelial growth factor (VEGF) therapy to control their neovascular age-related macular degeneration (AMD) revealed that adjunctive low-voltage, external beam X-ray irradiation reduced the frequency of anti-VEGF re-treatment. ${ }^{1}$ Participants in the IRay Plus Anti-VEGF Treatment For Patients With Wet AMD (INTREPID) Study who received 16 Gy or 24 Gy stereotactic radiotherapy (SRT) had a $25 \%$ reduction in the number of intravitreal injections compared with the sham arm over a 2-year period. ${ }^{2}$ Other clinical trials combining epimacular brachytherapy (that required vitrectomy with delivery of $\beta$ radiation to the macula) from a strontium source with antiVEGF treatments found neither visual benefit nor a reduced need for re-treatments when compared with anti-VEGF monotherapy. ${ }^{3}$

Ionising radiation has the potential to harm retinal vessels, with the damage becoming manifest many years after exposure, a condition termed radiation retinopathy (RR). ${ }^{4} 5$ Because the IRay device delivers precisely targeted radiotherapy and the area of retinal exposure is extremely small, we did not expect florid RR to develop. However subtle microvascular damage is difficult to differentiate from vascular abnormalities that occur in the context of neovascular AMD, ${ }^{6}$ therefore we designed a robust grading system to monitor signs of incident microvascular abnormalities (MVAs), and the present report details the clinical features and incidence of MVAs that occur in association with SRT.

\section{Participants and methods}

Details of the INTREPID Study have been reported. ${ }^{1}$ Briefly, 230 participants with neovascular AMD were recruited into a randomised, double-masked, sham-controlled clinical trial, across 21 European sites (Clinical Trial Registration at www.clinicaltrials.gov, identifier: NCT01016873; accessed 21 December 2014). Eligibility criteria were a confirmed diagnosis of neovascular AMD in the study eye with at least three intravitreal antiVEGF injections in the year preceding enrolment, and confirmation by the investigator of the need for ongoing anti-VEGF treatments evidenced by increased fluid or persistent cysts on optical coherence tomography (OCT), or leakage on fluorescein angiography (FA). The full list of inclusion and exclusion criteria has already been reported. ${ }^{1}$ Participants were randomised to 16 Gy or 24 Gy of SRT with as needed anti-VEGF therapy (SRT group) or sham SRT with as needed anti-VEGF therapy (anti-VEGF monotherapy group). All participants provided written informed consent, and the trial complied with the tenets of the Declaration of Helsinki.

Based on the existing knowledge of the radiobiology of retinal irradiation, ${ }^{7-9}$ the total dose 


\begin{tabular}{|c|c|c|}
\hline Imaging modality & Feature & Description \\
\hline \multirow[t]{2}{*}{ Colour photograph } & Nerve fibre layer infarcts & Cotton wool spots \\
\hline & Retinal haemorrhage & $\begin{array}{l}\text { Flecks of haemorrhage assuming } \\
\text { a feathery or circular disposition } \\
\text { indicating nerve fibre layer location } \\
\text { or in the inner retina. }\end{array}$ \\
\hline \multirow[t]{6}{*}{$\begin{array}{l}\text { Fluorescein } \\
\text { angiogram }\end{array}$} & Microaneurysms & $\begin{array}{l}\text { Focal circular outpouchings from } \\
\text { retinal microvessels }\end{array}$ \\
\hline & Luminal irregularities & $\begin{array}{l}\text { Segmental narrowing of retinal } \\
\text { vessels }\end{array}$ \\
\hline & $\begin{array}{l}\text { Retinal vascular } \\
\text { telangiectasia }\end{array}$ & $\begin{array}{l}\text { Fusiform dilations of retinal } \\
\text { microvessels }\end{array}$ \\
\hline & Capillary non-perfusion & $\begin{array}{l}\text { Areas of decreased fluorescence in a } \\
\text { perivascular distribution }\end{array}$ \\
\hline & Vascular sheathing & $\begin{array}{l}\text { Glistening or white perivascular } \\
\text { appearance indicating presence of } \\
\text { gliosis/fibrosis }\end{array}$ \\
\hline & $\begin{array}{l}\text { Leakage from retinal } \\
\text { vessels }\end{array}$ & $\begin{array}{l}\text { Diffuse staining and } \\
\text { hyperfluorescence along the length } \\
\text { of retinal vessels }\end{array}$ \\
\hline $\begin{array}{l}\text { Optical coherence } \\
\text { tomography }\end{array}$ & Intraretinal oedema & $\begin{array}{l}\text { Hyporeflective areas within the } \\
\text { neurosensory retina in an area not } \\
\text { occupied by the neovascular lesion }\end{array}$ \\
\hline
\end{tabular}

delivered during SRT (24 Gy or less), and the small focal dose distribution, it was anticipated that radiation-induced MVAs would be mild and possibly transient. Therefore, the monitoring regime of participants in the INTREPID trial was specifically tailored to identify subtle changes.

These included a clinical biomicroscopic examination of the eye at every visit with rigorous recording of fundus features, imaging to standardised protocols for colour fundus photography, OCT 6 monthly and FA annually. Because RR can occur after many years, a week 156 safety assessment was performed, to document the retinal status using all three imaging modalities.

Reading centre graders were trained to detect and record MVA, and to distinguish these from AMD-related vascular changes. Graders scrutinised the en face imaging modalities namely colour and FA for features of MVA and also simultaneously reviewed the radial B scans which were captured centred on the geometrical centre of the fovea. The $3 \times 3$ OCT radial star pattern scans covered an area of the retina that was smaller than the $35^{\circ}$ photographic field on colour and FA images.

Table 1 defines the microangiography changes detected by each imaging modality. Microaneurysms, vascular sheathing, luminal irregularities, telangiectatic vessels and perivascular capillary dropout were graded on the early frames (15 s to $30 \mathrm{~s}$ ) of the FA. Graders recorded these features regardless of whether they were present within the boundaries of the neovascular AMD lesion or beyond it and localised them to the quadrants of the retina. If any of the features described in table 1 were noted (primary detection), the study sponsor was notified. A second level arbitration of whether the MVAs were consistent with the natural history of neovascular AMD or related to SRT exposure was performed by the reading centre clinician. Change in severity of MVA was based on increasing or decreasing area of involvement and the appearance or disappearance of features of MVA. The images of all cases that were identified by graders regardless of the outcome of arbitration by the clinician arbitrator were examined by two retina experts (JS and UC) who independently assigned relatedness to SRT or not. In the event of
Table 2 Type, frequency and earliest appearance of microvascular abnormalities (MVAs) identified after radiotherapy in years 1 and 2

\begin{tabular}{|c|c|c|c|}
\hline Image modality & Feature & $\begin{array}{l}\text { Cumulative } \\
\text { frequency } \\
\text { M } 18 \text { and } M 24 \\
\text { (N=18 eyes) }\end{array}$ & $\begin{array}{l}\text { Earliest } \\
\text { appearance } \\
\text { (months) }\end{array}$ \\
\hline \multirow[b]{2}{*}{ Colour photograph } & Nerve fibre infarct & 6 & 12 \\
\hline & Retinal haemorrhage & 10 & 18 \\
\hline \multirow{7}{*}{$\begin{array}{l}\text { Fluorescein } \\
\text { angiogram }\end{array}$} & Microaneurysms & 7 & 24 \\
\hline & Luminal irregularities & 13 & 24 \\
\hline & Telangiectatic vessels & 8 & 24 \\
\hline & $\begin{array}{l}\text { Perivascular capillary } \\
\text { dropout }\end{array}$ & 15 & 24 \\
\hline & Vascular sheathing & 10 & 24 \\
\hline & Vascular staining & 15 & 24 \\
\hline & $\begin{array}{l}\text { Mean area of MVA } \\
\text { involvement }\end{array}$ & $3.2 \pm 3.0$ (SD) $\mathrm{mm}^{2}$ & \\
\hline $\begin{array}{l}\text { Optical coherence } \\
\text { tomography }\end{array}$ & $\begin{array}{l}\text { Intraretinal oedema in } \\
\text { the area of MVA }\end{array}$ & $1^{*}$ & 24 \\
\hline
\end{tabular}

The area of retina involved by MVA was measured on an early fluorescein angiographic frame.

*The OCT scan lines covered the area of MVA in only one eye.

disagreement between the experts a third expert (SM) performed further adjudication.

The incidence of MVA was examined using Kaplan-Meier methods. The exact date of the event was not known, so this was considered to be the visit date. Patients were censored on their last visit date if they had not had an event prior to exiting the study.

\section{RESULTS}

Apart from two occasions where features suggestive of radiation-induced microangiopathy were noted, clinical examination did not result in the identification of MVAs. MVAs reported by the reading centre are shown in table 2 , along with their cumulative frequency of detection by imaging modality and time of appearance. The imaging modality which best showed the region of retina involved by MVA was FA which revealed the telangiectatic and dilated microvessels most clearly and area measurements were based on this modality (table 2). MVA that were observed on FA included isolated microaneurysms, luminal irregularities, focal areas of capillary closure that were typically located adjacent to the second order retinal arterioles, distended (bullous) arteriolar and venular tips, telangiectatic segments and late staining of the vessel walls. Vascular luminal irregularities and perivascular capillary dropout were the most commonly encountered angiographic features. When MVAs were present, they usually occurred in the region between the centre of the macula and the optic disk and more often in the inferior macula. OCT using the star pattern scan which was popular at the time when the INTREPID Study was conducted was not useful in understanding the features of MVA. This was because the star pattern B scans rarely, if ever, transacted the region of the retina involved by the MVA in most cases. Of the 18 eyes with MVA, foveal involvement (defined as the presence of MVA within the central zone of the ETDRS grid) occurred in 8. MVAs were multifocal in six eyes (ie, distinct and separate regions of the retina exhibited MVA). In three of these there was foveal involvement.

Table 3 shows the frequency of occurrence of MVAs as determined by the reading centre by arm of study, the assignment 
Table 3 Microvascular abnormalities (MVAs) by arm of study and expert adjudication of relatedness of visual acuity loss

\begin{tabular}{|llll}
\hline BASELINE & $\begin{array}{l}\text { Monotherapy } \\
(\mathrm{N}=80)\end{array}$ & $\begin{array}{l}16 \mathrm{~Gy} \\
(\mathrm{~N}=75)\end{array}$ & $\begin{array}{l}24 \mathrm{~Gy} \\
(\mathrm{~N}=75)\end{array}$ \\
\hline MVA called by reading centre & 0 & 1 & 1 \\
\hline MVA confirmed by independent experts & 0 & 0 & 0 \\
\hline YEAR 1 & $\begin{array}{l}\text { Monotherapy } \\
(\mathrm{N}=79)\end{array}$ & $\begin{array}{l}16 \mathrm{~Gy} \\
(\mathrm{~N}=74)\end{array}$ & $\begin{array}{l}24 \mathrm{~Gy} \\
(\mathrm{~N}=72)\end{array}$ \\
\hline MVA called by reading centre & 1 & 2 & 4 \\
\hline MVA confirmed by independent experts & 0 & 0 & 0 \\
\hline Vision loss associated with RT & 0 & 0 & 0 \\
\hline YEAR 2 & Monotherapy & $16 \mathrm{~Gy}$ & $24 \mathrm{~Gy}$ \\
& $(\mathrm{~N}=73)$ & $(\mathrm{N}=69)$ & $(\mathrm{N}=68)$ \\
\hline MVA called by reading centre & 6 & 11 & 13 \\
\hline MVA confirmed by independent experts & 0 & 9 & 9 \\
\hline Vision loss associated with RT & 0 & 0 & 2 \\
\hline YEAR 3 & Monotherapy & $16 \mathrm{~Gy}$ & $24 \mathrm{~Gy}$ \\
& $(\mathrm{~N}=67)$ & $(\mathrm{N}=58)$ & $(\mathrm{N}=64)$ \\
\hline MVA called by reading centre & 1 & 22 & 19 \\
\hline MVA confirmed by independent experts & 0 & 19 & 18 \\
\hline Eyes with MVA regression & 0 & 3 & 6 \\
\hline Vision loss associated with SRT & 0 & 4 & 1 \\
\hline
\end{tabular}

Table 3 the accrual of microvascular abnormalities (MVA) by year. The frequency of MVA from the reading centre grading and that after expert consensus adjudication are shown. The number of eyes with VA loss possibly attributable to MVA in the opinion of the experts is also shown.

MVA, microvascular abnormality; Gy gray; SRT, stereotactic radiotherapy. of MVA by relatedness to SRT by the experts, and whether VA loss was, at least in part, attributable to the presence of MVA. MVA was $<2 \%$ in the anti-VEGF monotherapy arm and $36 \%$ in the two SRT arms. There was a suggestion of a dose effect with more cases of MVA reported in the 24 Gy arm compared with the $16 \mathrm{~Gy}$. After expert verification, the frequency of MVA in the combined SRT-exposed arms was $0 \%$ in year $1,13.1 \%$ in year 2 and $30.3 \%$ in year 3 . The Kaplan-Meier graphs (figures 1 and 2) show the frequency of development of MVA in the three study arms over time. In figure 1 all reading centre grader callouts of MVA are included and in figure 2 only those identified after expert consensus of SRT relatedness. Expert classification of MVA related to SRT showed no false-positive identifications in the anti-VEGF monotherapy arm.

In year 1 there was no case of vision loss attributed to SRT-related MVA. In year 2 the location of SRT-induced MVA was such that it may have contributed to vision loss in 2 of 18 cases, with the proportion remaining similar (5 of 37 cases) in year 3 . To examine the effect of the presence of MVA on change in visual acuity from baseline to 24 months, we computed this change in the control group (anti-VEGF monotherapy only), the entire group exposed to SRT (16 Gy and 24 Gy combined), and for two subsets of those exposed to SRT, namely those with or without MVA. Mean visual acuity was similar across all four comparisons suggesting that SRT-induced MVA did not modify visual outcome. $^{2}$ Likewise at 36 months mean visual acuity remained similar across the four groups, although formal statistics were not performed (figure 3).

\section{KM Analysis of MVA (by Reading Centre) - CLHOO2}

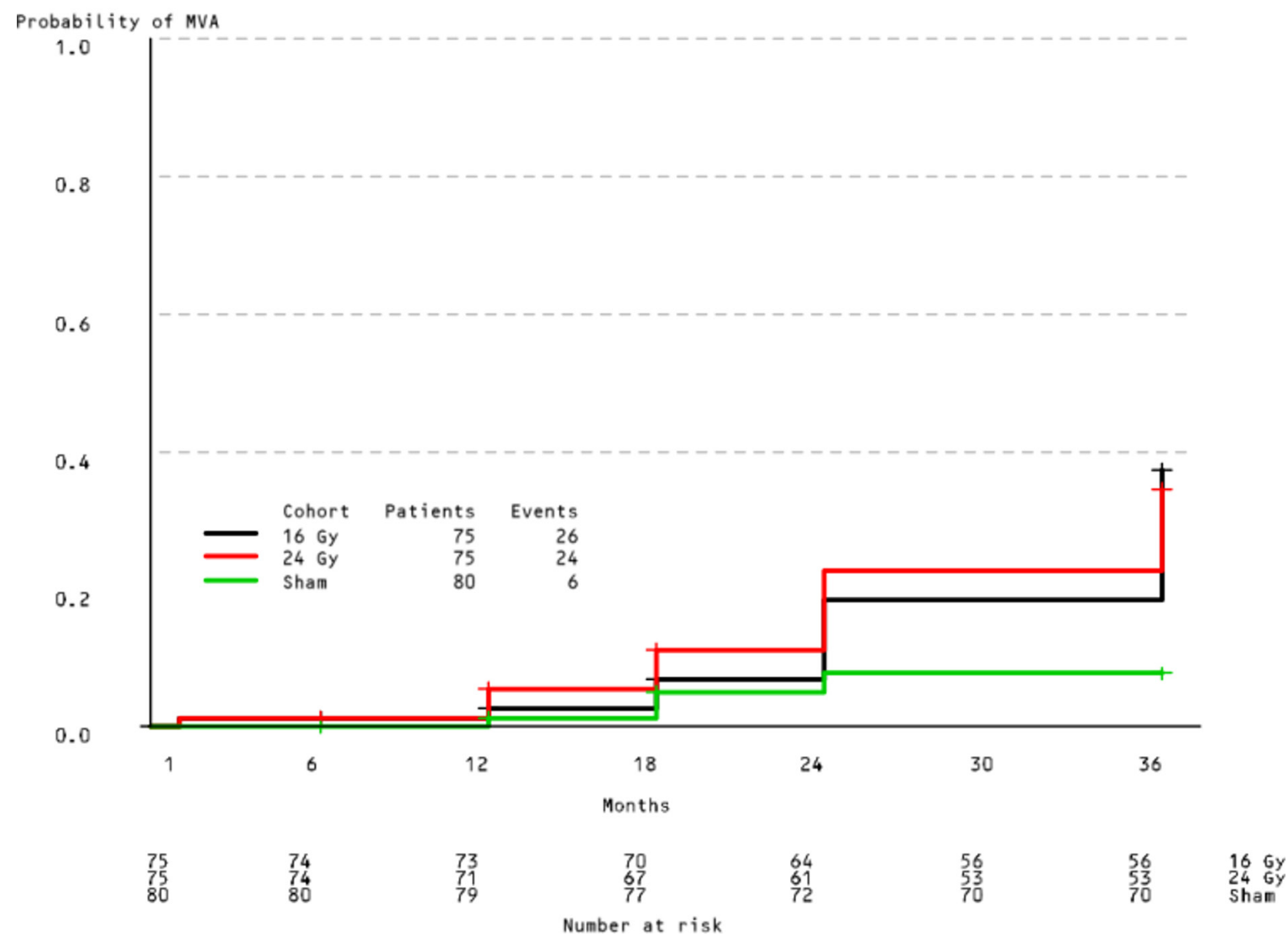

Figure 1 The Kaplan-Meier graph shows the rate of development of microvascular abnormalities (MVAs) by arm of study as reported by the reading centre. The numbers at risk reduce as the data become censored. 
KM Analysis of MVA (by Independent Experts) - CLHOO2

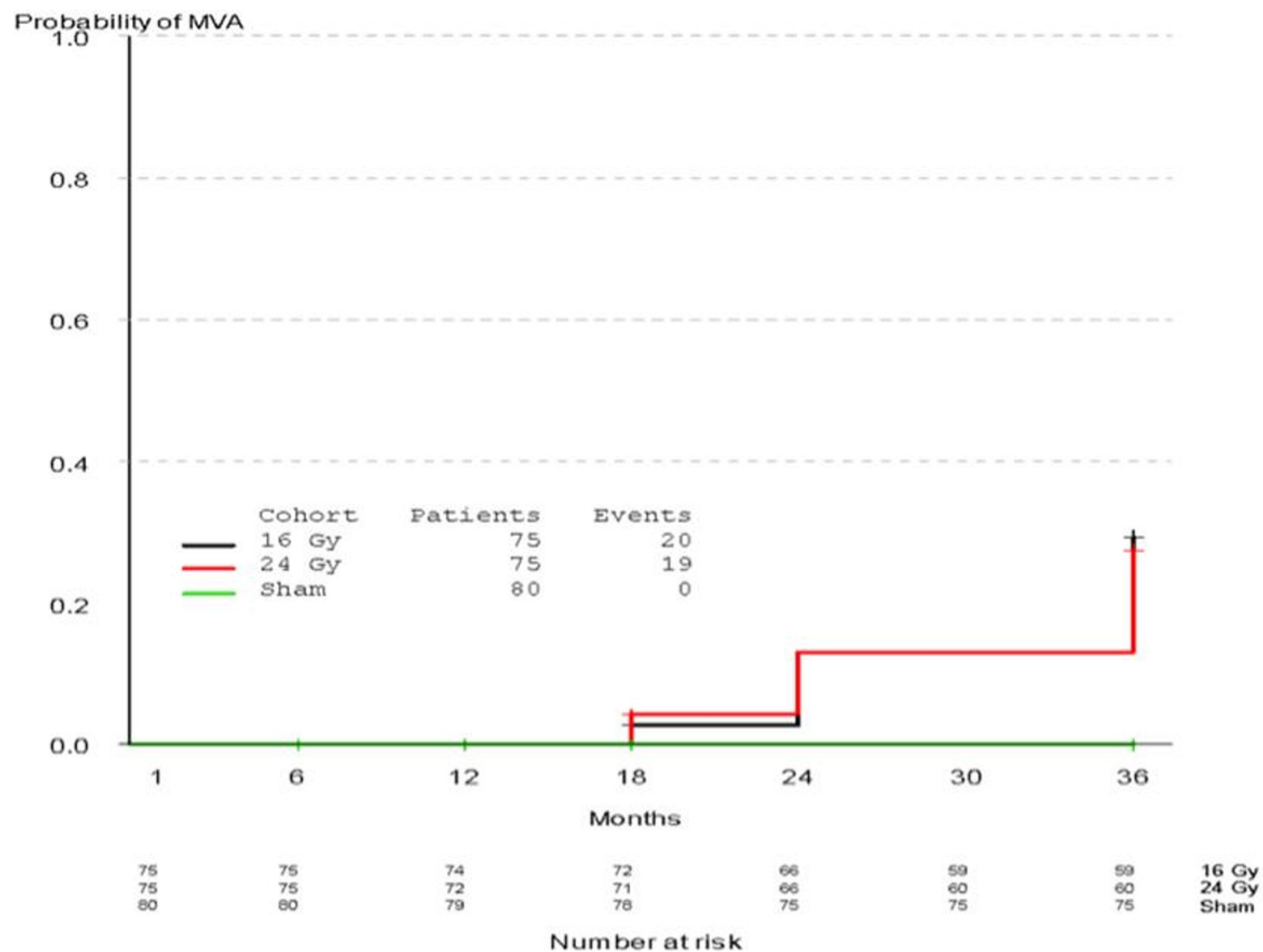

Figure 2 The Kaplan-Meier graph shows the rate of development of microvascular abnormalities (MVAs) by arm of study after expert adjudication. The numbers at risk reduce as the data become censored.

In 9 of the 18 eyes exhibiting MVA at 24 months, the vessels normalised and leakage decreased. In four cases complete resolution of the MVAs was noted. The mean area of retina occupied by MVA in all eyes with MVA at month 24 was $3.5 \mathrm{~mm}^{2} \pm 0.9$ (SD). This reduced to $2.35 \mathrm{~mm}^{2} \pm 1.4$ by month 36 .

Figure 4 shows an eye exposed to 24 Gy of SRT, demonstrating capillary non-perfusion and telangiectasia, with the area of involvement reducing over time.

\section{DISCUSSION}

The INTREPID Study was a 2-year randomised, sham-controlled clinical trial of combination SRT with anti-VEGF therapy versus anti-VEGF monotherapy that showed a reduction in the need for re-treatment with anti-VEGF in the adjunctive therapy arm. ${ }^{1}$ The present report describes the development of retinal MVA in eyes exposed to 16 Gy or 24 Gy ionising radiation. Focal areas of MVA attributable to SRT were observed in approximately a quarter to a third of eyes by year 3 . The average visual outcomes were similar comparing SRT-treated eyes with or without MVAs to each other, or control eyes. The lack of visual damage in most participants is probably because the area of retinal involvement by MVA was small (average of approximately 1.5-2 disc areas), and most occurred outside the fovea. However, in a small number of participants (two cases in year 2 and five cases in year 3) central macular involvement was judged to be present and contributing to vision loss.
MVAs were rare in the first year after SRT. At the month 18 visit cotton wool spots and or focal areas of intraretinal haemorrhage were observed in colour images but as FA was not part of the protocol for this visit it was not possible to document angiographic features. At the month 24 FA, microvascular luminal irregularities were the most commonly observed feature along with perivascular capillary drop out. The MVAs had a predilection for the area inferotemporal to the optic disk. Examination of the SRT treatment plan, isodose distribution curves, ocular anatomy and treatment protocol did not reveal any reasons for this finding. FA was the optimum modality for the detection of MVA as the high-resolution dynamic nature of this technology allowed identification of abnormal morphology in the microvasculature of the retina suggesting that follow-up should include FA as a monitoring strategy. However, with the advent of OCT A, a newer technology which yields high resolution non-invasively and rapidly acquired images of the central retinal field, this might be the imaging modality of choice in the follow-up of patients whose retinas have been exposed to ionising radiation.. ${ }^{1011}$

Recently it was reported by Jackson et al that MVAs occur in about $14 \%$ of eyes as part of the natural history of neovascular AMD, and that it may be difficult to distinguish these from radiation-induced MVAs. ${ }^{6}$ The same investigators also stated that CWS and capillary non-perfusion were generally not observed in neovascular AMD eyes and that these signs may be 


\section{Change in Visual Acuity at 36 Months}

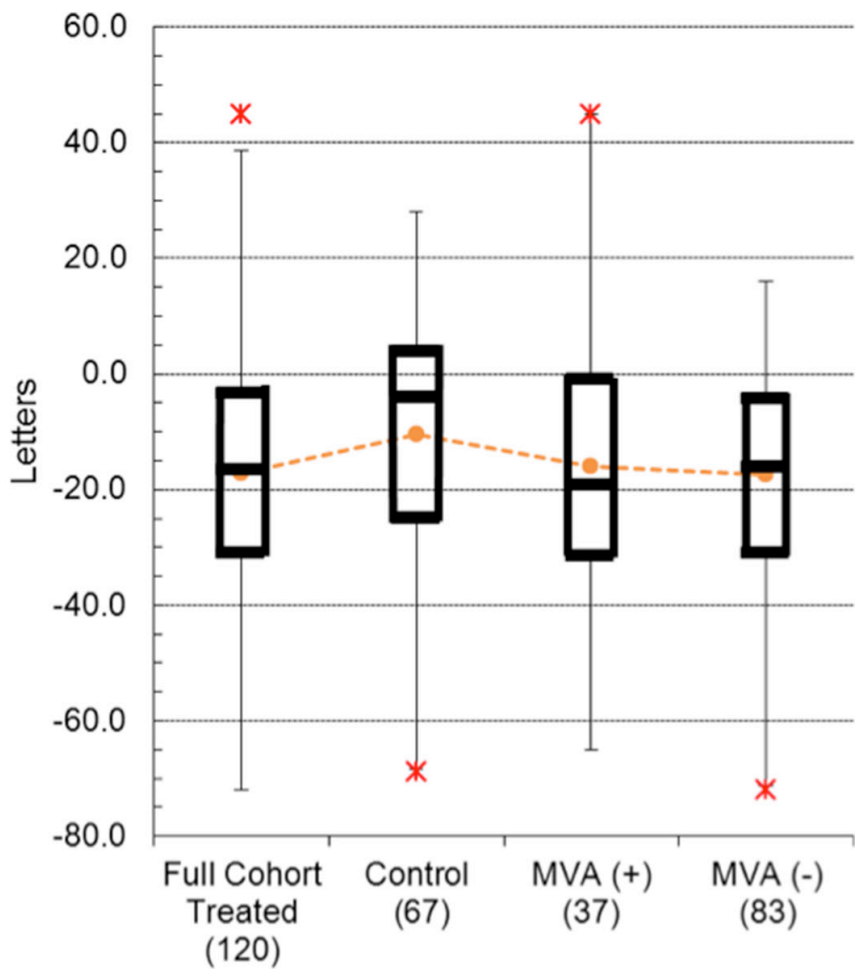

Figure 3 Box plot showing change in mean visual acuity at month 36. From left to right the first two groups are the entire RT-treated cohort (16 Gy and $24 \mathrm{~Gy}$ ) and the sham RT-treated (control). The subsequent two box plots are the RT-treated cohort split by MVA present $(n=37)$ and MVA absent $(n=83)$. The mean VA is similar across the four groups.

more suggestive of radiation exposure, or other diseases. ${ }^{6}$ The present study however showed that SRT-related MVAs can be distinguished with a high degree of reliability from neovascular AMD-associated retinal vessel abnormalities by employing a systematic approach, as the SRT-induced disease was characteristically segmental, with distinct angiographic appearances, and localised to areas of the fundus remote to the neovascular AMD lesion.

An important reason for the disparity between the present study and that of Jackson et $a l^{6}$ is that the latter was a retrospective analysis of images collected as part of another clinical trial reporting all non-specific retinal vascular changes that are present in eyes with neovascular AMD. The present study, on the other hand, had defined the specific features of radiation-induced damage and constructed the grading protocol with this aim in mind prior to the onset of the INTREPID trial. Also, the absence of such features in the sham SRT-exposed eyes in the present study was in distinct contrast to SRT-exposed eyes and detection of MVA was negligible in the former throughout the duration of the study.

In the eye there is a relatively radioresistant neuropile and a radiosensitive mesenchyme. ${ }^{12}$ This differential in radiosensitivity between the vascular endothelium and the neuropile had prompted researchers to undertake a clinical trial using fractionated external beam radiation as a treatment of neovascular AMD. ${ }^{13}$ However, the standard of care for neovascular AMD moved from laser-based therapies to biologicals, and antiVEGF agents were shown to result in improved outcomes when injected into the eye. ${ }^{14} 15$ These treatments come at huge cost, require invasive and repeated administration usually on a 4-8 weekly cycle, and despite treatment the long-term visual gain is hampered by fibrosis limiting visual recovery. ${ }^{1617}$ Thus there was resurgence in interest in radiotherapy as an adjunctive. Balanced against this potential for benefit is the knowledge that adding radiotherapy could itself result in the microangiopathy of the retina, induced by exposure to ionising rays.

The severity and onset of RR varies markedly depending on total dose, fractionation and the type of radiation (teletherapy or brachytherapy). ${ }^{18}$ In addition, the tissue volume irradiated, the concurrent administration of chemotherapeutic agents and pre-existing disease such as hypertension and diabetes also influence the development of RR. ${ }^{18}$ In INTREPID a single dose of 16 Gy or 24 Gy was chosen as the target was non-neoplastic; mesenchymal structures such as vascular malformations respond better to smaller doses of larger fraction sizes when compared with tumour eradication strategies. ${ }^{19}$ Furthermore, the literature
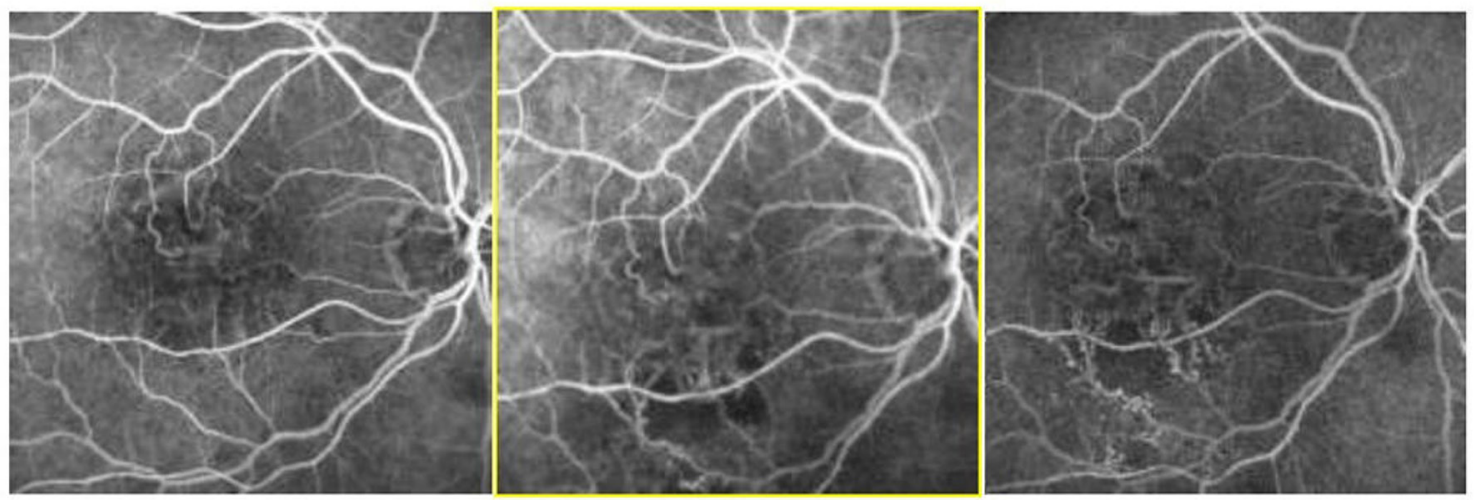

Figure 4 The set of angiographic frames shows the evolution of microvascular abnormalities from a single participant in the INTREPID trial exposed to $24 \mathrm{~Gy}$ of radiation. The frames are from the month 12, month 24 and month 36 visits in sequence from left to right. The foveal microcirculation from the angiographic frame at the 12-month visit shows a retinal angiomatous proliferation in the superior part of the macula (arrow) and which remains visible in the 24-month and 36-month visits. In the month 24 image there are several areas of retinal capillary non-perfusion, microaneurysms and segmental irregularities of the vessel lumina (telangiectasia) occupying approximately two disc areas. At month 36 the telangiectatic segments had reduced and the areas of capillary non-perfusion had decreased in both size and number. 
on patients where the entire eye was exposed to radiotherapy found that when the total dose is below $16 \mathrm{~Gy}$, fraction sizes of less than 2.0 Gy do not develop significant RR. ${ }^{20}{ }^{21}$ This study corroborates this outcome, and shows that a single dose of low energy, external beam X-ray radiation up to $24 \mathrm{~Gy}$ with a narrow $4 \mathrm{~mm}$ diameter isodose centred on the fovea does not result in clinically significant RR.

Strengths of the present study include its prospective design, prespecified safety grading outcomes and the robust reporting of MVA with multilevel checks to assign relatedness to SRT. An important weakness is the absence of data beyond 3 years and the loss to follow-up in year 3. Also it is possible that severe scarring in the central macula could have obscured some of the more subtle cases of MVA. Therefore the true incidence of MVA attributable to RT, both during the trial and beyond year 3 , remains unknown.

In summary, the present study shows the development of mild SRT-induced MVA and that these are distinguishable from non-specific retinal vessel abnormalities that may be encountered in eyes with chronic neovascular AMD. For most patients, SRT-induced MVAs did not impact on visual function and spontaneous improvement in microvascular morphology was also observed in some cases. Based on the appearance, area of involvement, localisation of the clinical and angiographic features, and the reversal of some of these features over time, the term RT-induced MVA is preferable to permit distinction from typical radiation retinopathy that is observed following exposure of large volumes of ocular tissue to high doses of ionising rays such as that used in oncological practice.

Acknowledgements We thank the sponsor's data management and statistical provider for their support in analysing the data. We also thank the site principal investigators and their research teams who participated in the INTREPID trial

Contributors TLJ, UC, DOS, AC were substantial contributors to the conception and design of the work. AM UC IS DO AC TLJ SM and LD were involved in the acquisition and interpretation of data. The analysis was undertaken by IDDI (LD) who were the sponsor's assigned data management and statistical provider . FJF TJ, SM and UC drafted the manuscript and all authors revised it for intellectual content. All authors gave final approval of the version published.

Funding This study was funded and sponsored by Oraya Therapeutics. The sponsor was responsible for the design and conduct of the study. The sponsor played no role in the development of the reading center grading protocols.

Competing interests UC and TLJ have received honoraria for attendance at advisory boards and have acted as consultants to Oraya therapeutics. AM has no conflicts to report. JS, FF and SM report compensations for their time. DOS and AC were employees of Oraya Therapeutics at the time of this study.

Patient consent Obtained.

Ethics approval NHS Research Ethics Committee, 10/NIR02/40.
Provenance-and-peer-review Not commissioned; externally peer reviewed.

(c) Author(s) (or their employer(s)) 2019. No commercial re-use. See rights and permissions. Published by BMJ.

\section{REFERENCES}

1 Jackson TL, Chakravarthy U, Kaiser PK, et al. Stereotactic radiotherapy for neovascular age-related macular degeneration: 52-week safety and efficacy results of the INTREPID study. Ophthalmology 2013;120:1893-900.

2 Jackson TL, Chakravarthy U, Slakter JS, et al. Stereotactic radiotherapy for neovascular age-related macular degeneration: year 2 results of the INTREPID study. Ophthalmology 2015;122:138-45.

3 Dugel PU, Bebchuk JD, Nau J, et al. Epimacular brachytherapy for neovascular age-related macular degeneration: a randomized, controlled trial (CABERNET). Ophthalmology 2013;120:317-27.

4 Parsons JT, Bova FJ, Fitzgerald CR, et al. Radiation retinopathy after externalbeam irradiation: analysis of time-dose factors. Int J Radiat Oncol Biol Phys 1994;30:765-73

5 Archer DB, Gardiner TA. Ionizing radiation and the retina. Curr Opin Ophthalmol 1994;5:59-65.

6 Jackson TL, Danis RP, Goldbaum M, et al. Retinal vascular abnormalities in neovascular age-related macular degeneration. Retina 2014;34:568-75.

7 Hall EJ. Time, Dose and Fractionation in radiotherapy, Chapter 22 in Radiobiology for the Radiologist. 5th edn. Philadelphia: Lippincott Williams \& Wilkins, 2000:387-418.

8 Brenner DJ. The linear-quadratic model is an appropriate methodology for determining isoeffective doses at large doses per fraction. Semin Radiat Oncol 2008;18:234-9.

9 Chakravarthy U, Gardiner TA, Archer DB, et al. A light microscopic and autoradiographic study of non-irradiated and irradiated ocular wounds. Curr Eye Res 1989;8:337-48.

10 Subhash HM, Leahy MJ. Microcirculation imaging based on full-range high-speed spectral domain correlation mapping optical coherence tomography. J Biomed Opt 2014;19:21103.

11 Jia Y, Bailey ST, Wilson DJ, et al. Quantitative optical coherence tomography angiography of choroidal neovascularization in age-related macular degeneration. Ophthalmology 2014;121:1435-44.

12 Archer DB. Doyne Lecture. Responses of retinal and choroidal vessels to ionising radiation. Eye 1993;7(Pt 1):1-13.

13 Hart PM, Chakravarthy U, Mackenzie G, et al. Visual outcomes in the subfoveal radiotherapy study: a randomized controlled trial of teletherapy for age-related macular degeneration. Arch Ophthalmol 2002;120:1029-38.

14 Brown DM, Kaiser PK, Michels M, et al. Ranibizumab versus verteporfin for neovascular age-related macular degeneration. N Engl J Med 2006;355:1432-44.

15 Rosenfeld PJ, Brown DM, Heier JS, et al. Ranibizumab for neovascular age-related macular degeneration. N Eng/ J Med 2006;355:1419-31.

16 Martin DF, Maguire MG, Fine SL, et al. . Ranibizumab and bevacizumab for treatment of neovascular age-related macular degeneration: two-year results. Ophthalmology 2012;119:1388-98.

17 Chakravarthy U, Harding SP, Rogers CA, et al. Alternative treatments to inhibit VEGF in age-related choroidal neovascularisation: 2-year findings of the IVAN randomised controlled trial. Lancet 2013;382:1258-67.

18 Amoaku WM, Archer DB. Fluorescein angiographic features, natural course and treatment of radiation retinopathy. Eye 1990;4 ( Pt 5:657-67.

19 Flickinger JC, Kondziolka D, Niranjan A, et al. Dose selection in stereotactic radiosurgery. Prog Neuro/ Surg 2007:20:28-42.

20 Brown GC, Shields JA, Sanborn G, et al. Radiation retinopathy. Ophthalmology 1982;89:1494-501.

21 Krebs IP, Krebs W, Merriam JC, et al. Radiation retinopathy: electron microscopy of retina and optic nerve. Histol Histopathol 1992;7:101-10. 